\title{
Thermal Characterization of Innovative Sustainable Building Materials from Wool Textile Fibers Waste
}

\author{
C. Rubino ${ }^{1}$, M. Bonet-Aracil ${ }^{2}$, S. Liuzzi ${ }^{1}$, F. Martellotta ${ }^{1}$, P. Stefanizzi ${ }^{1}{ }^{*}$ \\ ${ }^{1}$ Politecnico di Bari, Dipartimento di Scienze dell'Ingegneria Civile e dell'Architettura, via Orabona 4, I70125 Bari, Italy \\ ${ }^{2}$ Grupo de Investigación en la Industria Textil (GIITEX). Departamento de Ingeniería Textil y Papelera, Universitat Politècnica \\ de València, Alcoy Alicante, España
}

Corresponding Author Email: pietro.stefanizzi@poliba.it

https://doi.org/10.18280/ti-ijes.632-423

Received: 15 February 2019

Accepted: 20 April 2019

\section{Keywords:}

wool fibers, natural binder, effective thermal

conductivity

\begin{abstract}
In the textile sector, fabrics produce waste from the earliest stages of production, particularly during the garment manufacturing process. The reuse of these materials as byproduct to create innovative building materials could be a solution to reduce the environmental impact and to limit the waste disposal costs. The use of chitosan as a natural binder was investigated in order to obtain more easily recyclable materials with a lower environmental impact. The physical and thermal performances were tested, studying the correlation between porosity and thermal parameters. The experimental thermal conductivity range was between 0.049 and $0.06 \mathrm{~W} \cdot \mathrm{m}^{-1} \cdot \mathrm{K}^{-1}$. Results proved the possibility to produce sustainable thermal insulation materials with performances comparable to conventional building products. Heat transfer through fibrous materials involves combined modes of heat transfer (conduction, convection, radiation) through the different phases (solid and gas): conduction in the solid and gas phase, and convection and radiative transfer through the gas phase. This paper focuses on the development and validation of a physical model for effective thermal conductivity in highly porous fibrous materials like wool panels with natural binder. If compared to the models currently available in the literature, the proposed model shows an improved correlation with the experimental data for the materials of interest.
\end{abstract}

\section{INTRODUCTION}

With the increasing demand of indoor comfort, the improvement of the thermal insulation of buildings is becoming the most effective way to get the reduction of heat losses through envelopes [1]. Buildings insulation is commonly realized using materials obtained from petrochemicals (polystyrene), or manufacturing processes which do not satisfy environmental sustainability (rock wool or glass wool) [2]. These materials cause negative impacts on the environment since their production stage (i.e. emissions during the raw material extraction or the manufacturing processes), up to their disposal stage, with several issues related to their reusing or recycling at the end of their life [3]. The growing awareness that the Earth has limited ability to absorb the impacts of human activities, encouraged several researchers to develop sustainable building materials by renewable energy sources and recycled raw materials [4]. The ecological properties of thermal insulating building products play an important role for architects and engineers in the selection process. The integration of eco-friendly materials in the building envelope has become the main passive prerequisite for ensuring indoor thermal comfort [5].

The reuse of waste generated from agricultural and industrial production activities offers an opportunity to both minimize the detrimental impacts of the construction sector on the environment, and the disposal-related issues [6].

Several researches on sustainable building insulation investigated on wastes originating from agricultural sector as natural raw resources in composite materials. Liuzzi et al. [7] showed the excellent hygrothermal performances of earthen plaster prepared by mixing clay and straw. Baccilieri et al. [8] studied the thermal performances of biocompatible insulating materials obtained from lime mixed with agricultural waste.

In recent decades, the use of textile waste in building materials has been gaining popularity due to the increase in their production following an escalation in consumptions [9]. Several authors have found that building composites produced from recycled textile materials have high thermal properties, are durable and environmentally friendly compared to the conventional ones. El Wazna et al. [10] and Patnaik et al. [11], provided a promising solution for buildings insulation based on needle punched nonwoven materials developed respectively from wool and acrylic waste or wool and polyester waste. The thermal insulation properties of these materials were measured in terms of the thermal conductivity and it was demonstrated that both type of mats could be considered suitable alternatives to traditional ones. Trajković et al. [12] focused on using polyester waste derived from the cutting processes of clothing industry to develop innovative panels usable as indoor and roofing insulation materials.

Whatever the manufacture process, textile composite materials are porous materials consisting of a solid matrix with an interconnected void. This structure determines their generally good heat transport properties [13]. The determination of the effective thermal conductivity of porous 
materials is very complex because it is dependent not only on the porosity, but also on the macroscopic structure of the materials. As a matter of fact, the way the solid components are connected developing a three-dimensional shape plays an important role [14]. The effective thermal conductivity of fibrous materials is the result of three modes of heat transfer: conduction in the solid and gaseous phase, convection in the gas, and radiation [15]. Tiliuoa et al. [16] carried out a thermal characterization of a fibrous insulation material made from recycled textile waste clothing for building applications. For the radiative properties evaluation, they adopted the inverse method based on the experimental measurements of hemispherical reflectance and transmittance. Schiavoni et al. [17] reported a state of the art of insulating materials used in the building sector comparing thermal performances, reaction to fire, and environmental properties. Tugnoli et al. [18] developed a physical model to calculate the effective thermal conductivity of fibrous fireproofing materials in typical operative conditions.

The present study investigates innovative insulating materials produced from textile waste of $100 \%$ Merino wool with a chitosan binder solution. One of the main advantages of wool over synthetic fibers is its biodegradability. In fact, natural fibers, thanks to their high nitrogen content, can decompose in about a year. In addition, the use of an organic binder like chitosan defines an even greener recycling process which makes the building industry more sustainable. Chitosan is a polysaccharide obtained by industrial treatment of chitin from crustacean shells. Recently, it has received more attention in the area of biomaterials and bio sourced materials thanks to its biodegradable, non-toxic and non-water soluble nature [19]. Martellotta et al. [20] developed innovative sound absorber panels obtained from olive pruning waste bonded by using a bio based chitosan glue. Mati-Baouche et al. [19] carried out a research to develop a new insulating bio-based composite made with chitosan and sun-flower's stalks particles.

The present paper focuses on the thermal characterization of innovative textile waste materials. The aim of the research is to better understand and quantify how the different heat transfer processes affect the effective thermal conductivity value of the investigated materials.

\subsection{Samples preparation}

A batting matrix of Merino wool was used to prepare the fibrous thermal insulation materials under investigation. The wool matrix was obtained by scouring and carding the textile waste discarded during the garment cutting process by a local clothing company.

A natural binder solution was obtained by preparing a chitosans solution $(15 \mathrm{~g} / \mathrm{L})$ with $5 \mathrm{~mL}$ of acetic acid.

Different samples obtained by combining the same percentages of binder and fibrous matrix (60\% and $40 \%$ respectively), but different density values (ranging between 80 and $197 \mathrm{~kg} \mathrm{~m}^{-3}$ ), were studied in order to characterize and compare their thermal behavior. The molding method was followed to produce the samples. Fibers were soaked in the chitosan solution and, before placing them in the molds, the excess amount of binder was eliminated by squeezing the liquid excess. The recycled textile fibers agglomerated quite homogeneously, entrapping air in the fibers network thus resulting in a potentially good insulating material. Finally, samples were dried in an oven at $100{ }^{\circ} \mathrm{C}$, for one hour.
For each density value, six cylindrical specimens, $5 \mathrm{~cm}$ thick, were prepared. Three of them had a $10 \mathrm{~cm}$ diameter in order to measure thermal properties and three had a $4 \mathrm{~cm}$ diameter to evaluate the porosity of the materials.
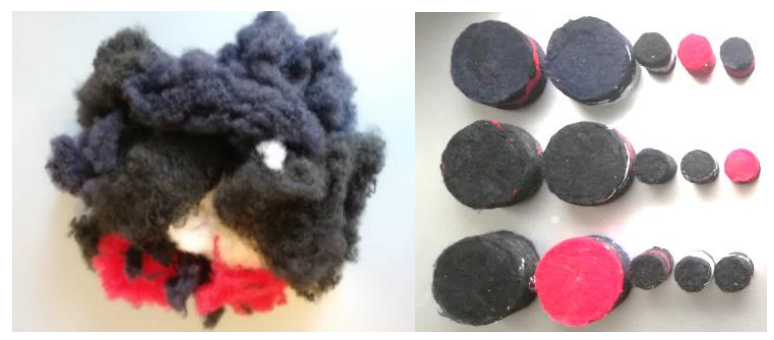

Figure 1. Samples of Merino wool

\subsection{Physical characterization}

ULTRAPYC 1200-e Quantachrome Helium gas Pycnometer was used to determine the porosity $\varepsilon$ :

$\varepsilon=1-\frac{\rho_{\text {bulk }}}{\rho_{\text {true }}}$

where $\rho_{\text {true }}$ and $\rho_{\text {bulk }}$ are the true and the bulk density of the materials, respectively.

Table 1. Properties of tested samples

\begin{tabular}{cccc}
\hline $\begin{array}{c}\text { Sample } \\
\text { code }\end{array}$ & $\begin{array}{c}\text { Bulk density, } \\
\boldsymbol{\rho}_{\text {bulk }}\end{array}$ & $\begin{array}{c}\text { True density, } \\
\boldsymbol{\rho}_{\text {true }}\end{array}$ & $\begin{array}{c}\text { Porosity, } \\
\boldsymbol{\varepsilon}\end{array}$ \\
\hline S1 & $197 \pm 1.7$ & $1336 \pm 19$ & $0.86 \pm 0.002$ \\
S2 & $145 \pm 1.6$ & $1326 \pm 7$ & $0.89 \pm 0.001$ \\
S3 & $122 \pm 1.2$ & $1326 \pm 14$ & $0.91 \pm 0.001$ \\
S4 & $80 \pm 1.0$ & $1282 \pm 23$ & $0.94 \pm 0.001$ \\
\hline
\end{tabular}

Table 1 compares the values of the bulk density and the true density and shows the porosity values for the tested materials. All values are given as mean value of five different measurements with the error representing the standard deviation of the mean.

The microstructural morphology of the woolen composite materials was characterized by a Scanning Electron Microscope (SEM) Phenom Fei TM.

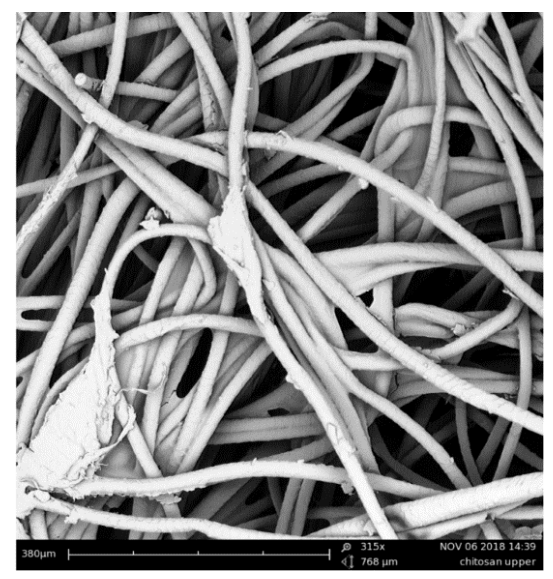

Figure 2. SEM imagine of fibrous insulation_315x

As it can be observed in Figure 2, the tested materials were composed of cylindrical fibers with a $20 \mu \mathrm{m}$ average diameter, randomly distributed. 


\section{THERMAL CHARACTERIZATION}

The thermal properties, i.e. thermal conductivity, thermal diffusivity and volumetric heat capacity, were experimentally evaluated by using the transient plane source device Isomet 2104 (Applied Precision Ltd). Measurement was based on the analysis of the temperature response of the tested material to heat flow impulses. The heat flow was induced by electrical heating using a resistor heater having a direct thermal contact with the surface of the sample. The advantage of this technique is the possibility to perform rapid measurements on relatively small samples.

Table 2. Thermal properties of tested samples

\begin{tabular}{ccccc}
\hline $\begin{array}{c}\text { Sampl } \\
\text { e code }\end{array}$ & $\begin{array}{c}\text { Thermal } \\
\text { conductivit } \\
\mathbf{y}, \boldsymbol{k}_{\boldsymbol{e}}\end{array}$ & $\begin{array}{c}\text { Thermal } \\
\text { diffusivity } \\
\boldsymbol{\alpha}\end{array}$ & $\begin{array}{c}\text { Specific } \\
\text { heat } \\
\text { capacity } \\
\boldsymbol{\boldsymbol { c }}\end{array}$ & $\begin{array}{c}\text { Mean } \\
\text { temperatur } \\
\mathbf{e}, \boldsymbol{T}_{\boldsymbol{m}}\end{array}$ \\
\hline S1 & 0.060 & $0.177 \times 10^{-6}$ & 1726 & 27.5 \\
S2 & 0.055 & $0.196 \times 10^{-6}$ & 1924 & 26.3 \\
S3 & 0.052 & $0.198 \times 10^{-6}$ & 2152 & 27.4 \\
S4 & 0.049 & $0.217 \times 10^{-6}$ & 2810 & 27.1 \\
\hline
\end{tabular}

The specific heat capacity values of tested materials were also calculated from volumetric heat capacity and density.

Table 2 shows the thermal properties of all tested samples.

The knowledge of $k_{e}$ values allows to compare the thermal efficiency of different building insulation materials [21]. In Figure 3, the thermal conductivity values of all woolen composite materials were plotted as a function of their density values, and a statistically significant $\left(\mathrm{R}^{2}=0.9972\right)$ linear correlation was found:

$k_{e}=9.79 \cdot 10^{-5} \rho+0.0405$

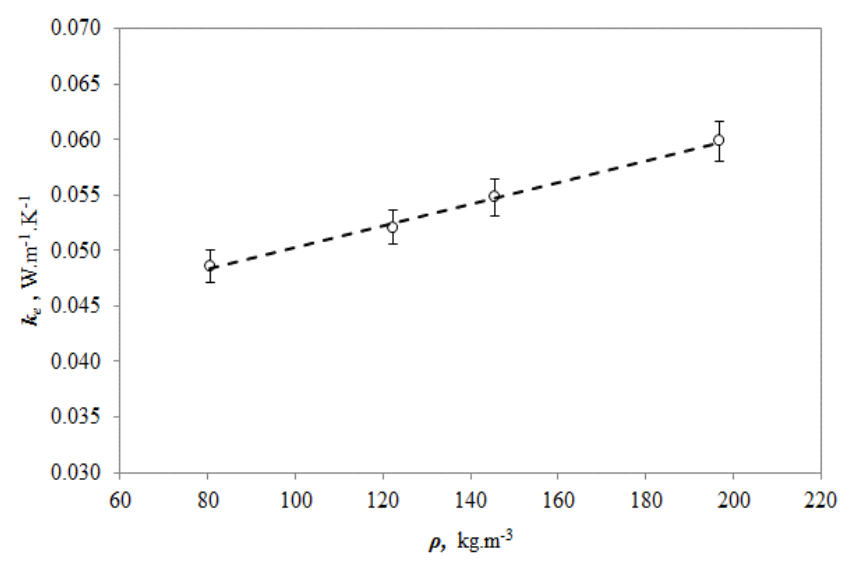

Figure 3. Thermal conductivity vs density

The highest $k_{e}$ value was associated to the material with the highest density. This could be due to the increase in fibrous matrix and the shrinking of voids due to the increase of the density value (Table 1). Thermal conductivity of a solid skeleton is higher than that of the air within the voids. Thus, the thermal conductivity value of the whole material increased [22]. The thermal conductivity range of the studied wool samples varied from 0.049 to $0.060 \mathrm{~W} \cdot \mathrm{m}^{-1} \cdot \mathrm{K}^{-1}$. As it can be observed in Table 3, the results were in agreement with the thermal performance exhibited by similar commercial materials and other natural fibrous materials (Figure 4).
Table 3. Comparison of thermal conductivity among similar insulation materials

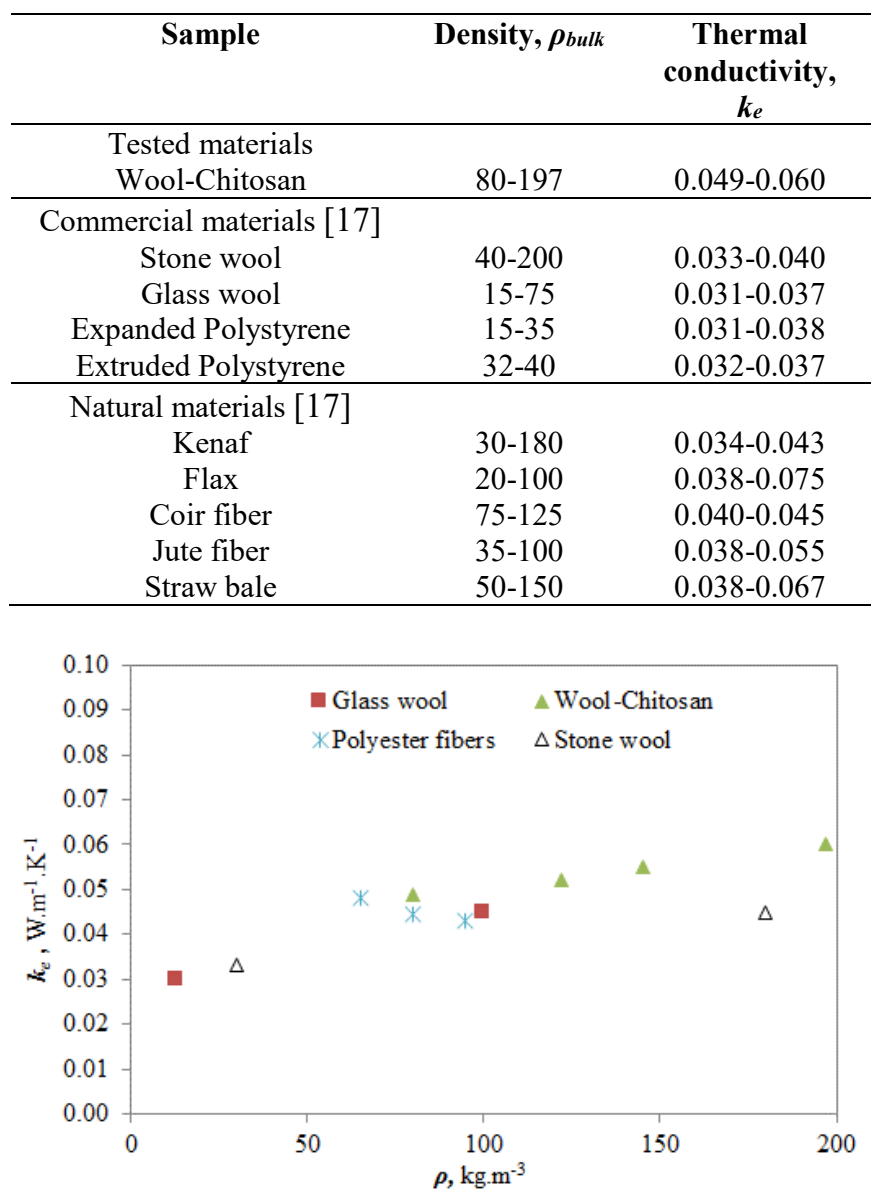

Figure 4. Thermal conductivity vs density for fibrous insulation materials

\section{CONDUCTIVE, CONVECTIVE AND RADIATIVE CONTRIBUTIONS}

The large majority of the building insulation materials belongs to the family of fibrous media, as the woolen composite investigated in the present study.

Fibrous materials are made of a solid matrix consisting of fibers and a gas phase formed by air. Therefore, the heat transfer can occur in three different ways: conduction $k_{c}$ through the fibrous solid matrix, and through the gas medium, i.e. the air trapped within the pores, natural convection $k_{c o n v}$ due to the movement of the air between fibers, and radiation $k_{r}$. Thus, the effective thermal conductivity $k_{e}$ can be represented as the sum of these contributions:

$k_{e}=k_{c}+k_{c o n v}+k_{r}=k_{s}+k_{g}+k_{c o n v}+k_{r}$

where $k_{s}$ and $k_{g}$ are the conduction terms due to solid fibers and gas, respectively.

The effective thermal conductivity within a fibrous material is one of the most difficult physical quantities to investigate, due to the influence of many factors such as the distance between fibers and their directions [16]. In this work, only conduction heat transfer, consisting of both solid and gaseous contribution, is considered. The choice of excluding convective and radiative contributions in this kind of highly 
porous materials can be justified by analyzing some appropriate dimensionless numbers, i.e. the modified Rayleigh number $R a^{*}$ and the Planck number $N$, which will be extensively discussed in the subsequent sections.

\subsection{Conduction: gas medium}

Gases in which the ratio of the mean molecular spacing and the mean molecular diameter is less than 7 can be described as being dense gases, conversely they can be considered dilute gases [23]. The rarefaction degree of air trapped in the pores of a porous material plays an important role to evaluate the contribution of the gas to heat conduction. In a dilute gas, the intermolecular forces can be neglected, and the molecules spend most of their time in free flight between successive collisions. In this aspect, the rarefaction effect in microsystems is attributed to the mean free path of the gas and it can be related to the Knudsen number $K n$. The Knudsen number is the characteristic dimensionless parameter for the theory of collisions and it is defined as the ratio of the mean free path of the gas molecules $\lambda$ to the characteristic length $\delta$ :

$K n=\frac{\lambda}{\delta}$

The mean free path $\lambda$ is the length that a molecule can travel before a collision event with a second molecule, and it is given by [24]:

$\lambda=\frac{k_{B} T}{\sqrt{2} P \pi d_{m}^{2}}$

where $k_{B}$ is the Boltzmann's constant, $T$ and $P$ are the temperature and the pressure of air and $d_{m}$ is the air collision diameter defined as the diameter of the circular area around an air particle in which the center of another particle must be for a collision to occur.

The characteristic length $\delta$ in a fibrous material is defined as the mean distance between the fibers and it can be calculated by an empirical formulation that assumes the fibers as a three-dimensional matrix [23]:

$\delta=\frac{3 \pi}{5} \frac{d}{f}$

where $d$ is the diameter of the fibers and $f=1-\varepsilon$ is the volume fraction of solid (being $\varepsilon$ the porosity of the samples).

Depending on the value of Knudsen number, different flow regimes take place. As $K n$ number increases, the degree of rarefaction becomes more significant and the continuum assumption breaks down [25]. The subdivision of the flow regimes is very important in order to choose the empirical model to apply for characterizing the gas heat conduction [18].

The Knudsen number was calculated and shown in Table 4 by assuming $d_{m}=0.369 \times 10^{-9} \mathrm{~m}$ at $293 \mathrm{~K}$. The Boltzmann's

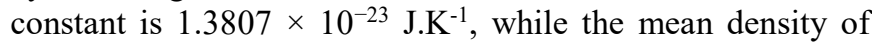
wool fibers was $1318 \mathrm{~kg} . \mathrm{m}^{-3}$ (Table 2) and their mean diameter, as obtained from the SEM imagine (Figure 1), was assumed to be approximately $20 \mu \mathrm{m}$.

As it can be observed, the Knudsen number was less than 0.01 . This means that the characteristic length was larger than the free mean path of the gas molecules. According to Zhang et al. [26], there was no slip between the wall of the pores and the air because thermal equilibrium between the pore walls and nearby fluid was achieved. Therefore, the fluid was treated in a continuum regime, dominated by intermolecular collisions, and was assumed $k_{g}=k_{\text {air }}=0.026 \mathrm{~W} \cdot \mathrm{m}^{-1} \cdot \mathrm{K}^{-1}$ [26].

Table 4. Mean free path, characteristic length and Knudsen number for all samples

\begin{tabular}{cccc}
\hline $\begin{array}{c}\text { Sample } \\
\text { code }\end{array}$ & Free path, $\boldsymbol{\lambda}$ & $\begin{array}{c}\text { Characteristic } \\
\text { length, } \boldsymbol{\delta}\end{array}$ & $\begin{array}{c}\text { Knudsen } \\
\text { number, } \boldsymbol{K} \boldsymbol{n}\end{array}$ \\
\hline S1 & $6.621 \times 10^{-8}$ & $2.522 \times 10^{-4}$ & $2.625 \times 10^{-4}$ \\
S2 & $6.621 \times 10^{-8}$ & $3.427 \times 10^{-4}$ & $1.932 \times 10^{-4}$ \\
S3 & $6.621 \times 10^{-8}$ & $4.073 \times 10^{-4}$ & $1.626 \times 10^{-4}$ \\
S4 & $6.621 \times 10^{-8}$ & $6.211 \times 10^{-4}$ & $1.066 \times 10^{-4}$ \\
\hline
\end{tabular}

\subsection{Convection: Gas medium}

According to [27], for natural convection to take place in a fibrous medium, the buoyancy forces must overcome viscous forces. Taking into account that the very small dimensions of the voids between the fibers make air movement negligible, heat transfer due to convection is likely to be insignificant and can be ignored without affecting accuracy. This assumption could be further justified by evaluating the modified Rayleigh number $R a^{*}$. According to Silbestrein [28], the sensibility of porous materials to convection is strongly linked to their air permeability. Consequently, the modified Rayleigh number $R a^{*}$ was calculated as follows:

$R a^{*}=\frac{g \beta \rho_{\text {air }}^{2} c_{\text {air }}}{\mu_{\text {air }}} \frac{K}{k_{e}} \Delta T S$

where $g$ is the gravity acceleration, $c_{\text {air }}, \beta, \rho_{\text {air }}$ and $\mu_{\text {air }}$ are respectively the specific heat capacity, volumetric thermal expansion coefficient, density, and dynamic viscosity of air; $K$, $k_{e}$ and $s$ are respectively permeability, thermal conductivity and thickness of the material and $\Delta T$ is the temperature difference between the two sides of the sample. If $R a^{*}$ is less than 40 , the convection effect can be neglected.

Table 5 shows the modified Rayleigh number calculated for all tested samples, by assuming $\Delta T=293 \mathrm{~K}$ and $s=0.05 \mathrm{~m}$. Thermal conductivity values resulted from experimental measurements (Table 2) and the values of air permeability were calculated according to the Davies's empirical correlation [39]:

$K=\frac{1}{16 f^{1.5}\left(1+56 f^{3}\right)} r^{2}$

where $f$ is the volume fraction of solid and $r$ is the mean radius of the fibers. Table 5 shows the values of air permeability for all tested samples.

Table 5. Air permeability and modified Rayleigh number for all tested samples

\begin{tabular}{ccc}
\hline $\begin{array}{c}\text { Sample } \\
\text { code }\end{array}$ & Air permeability, $\boldsymbol{K}$ & $\begin{array}{c}\text { Modified } \\
\text { Rayleigh } \\
\text { number, } \boldsymbol{R} \boldsymbol{a} *\end{array}$ \\
\hline S1 & $1.070 \times 10^{-5}$ & 0.5 \\
S2 & $1.782 \times 10^{-5}$ & 0.9 \\
S3 & $2.403 \times 10^{-5}$ & 1.3 \\
S4 & $4.475 \times 10^{-5}$ & 2.6 \\
\hline
\end{tabular}

As can be observed, the modified Rayleigh number varies from 0.5 (sample with highest density value) to 2.6 (sample with lowest density value), thus in all the cases convection may be neglected. 


\subsection{Radiation}

Thermal radiation heat transfer that takes place between two distant bodies depends on the difference of the fourth power of their absolute temperatures [30]. Therefore, the radiative contribution of the fibers in an insulating material may be particularly significant at high temperatures, while it can be neglected at temperatures around $293 \mathrm{~K}$. According to Lux et al. [31], the importance of radiative contribution could be evaluated by calculating the Planck number $N$ :

$N=\frac{k_{e}}{4 n^{2} \sigma T^{3} l_{p}}$

where $k_{e}$ is the experimental thermal conductivity, $n$ is the refractive index of air, $\sigma$ is the Stefan-Boltzmann's constant, $T$ is the temperature and $l_{p}$ is the mean free path of photons. When $N>10$, the contribution of radiation can be neglected.

The Planck number was calculated for all the samples, by assuming the mean free path of photons equivalent to the mean-fiber distance $\delta$, previously computed (Table 6).

Table 6. Planck number of tested samples

\begin{tabular}{cc}
\hline Sample code & Planck number, $\boldsymbol{N}$ \\
\hline S1 & 92 \\
S2 & 62 \\
S3 & 49 \\
S4 & 30 \\
\hline
\end{tabular}

As was expected, the Planck number assumed the maximum value $(\mathrm{N}=92)$ for the sample $\mathrm{S} 1$ with highest density value; and the minimum value $(\mathrm{N}=30)$ for the sample $\mathrm{S} 4$ characterized by lowest density. This means that the contribution of radiation can be neglected for all samples.

\subsection{Effective conductivity}

As a result of the negligible convective and radiative contribution to the effective conductivity, the heat transfer in the fibrous woolen materials under test was fundamentally due to conduction in solid and gas phases. Gong et al. [32] proposed a novel effective medium theory for modeling the thermal conductivity of porous materials. Five basic structural models, including the Series, Parallel, Maxwell-Eucken (two forms) and EMT models, for a two-component system were unified in a single equation:

$$
(1-\varepsilon) \frac{k_{s}-k_{e}}{k_{s}+2 k_{m}}+\varepsilon \frac{k_{g}-k_{e}}{k_{g}+2 k_{m}}=0
$$

where $k_{s}$ and $k_{g}$ are the conduction due to solid fibers and gas, respectively; $k_{m}$ is a variable value.

The equation can adapt to various models by simply changing values of $k_{m}$. When $k_{m}=k_{e}$, the above equation is the EMT model. When $k_{m}=k_{s}$, (or $k_{m}=k_{a}$ ), the equation is the Maxwell-Eucken (two forms, respectively). When $k_{m}=0$ the equation is the Series model, and when $k_{m}=\infty$ the equation is the Parallel model.

Considering sample S2, a value of $k_{s}=0.290 \mathrm{~W} \cdot \mathrm{m}^{-1} \cdot \mathrm{K}^{-1}$ was estimated by exploring several values for $k_{m}$ in order to identify which value minimizes the standard deviation between the experimental values $\left(k_{e}\right)$ and those estimated by the model $\left(k_{e, \text { est }}\right)$.

The best agreement between the model and the experimental $k_{e}$ values was obtained when $k_{m} \rightarrow \infty$, so that the equation (10) became:

$k_{e}=(1-\varepsilon) k_{s}+\varepsilon k_{g}$

Comparing the estimated effective conductivity $k_{e, e s t}$ yielded by Eq. (11), with the measured one $k_{e}$ the relative error $\frac{k_{e}-k_{e, e s t}}{k_{e}} \times 100$ was computed and given in Figure 5 .

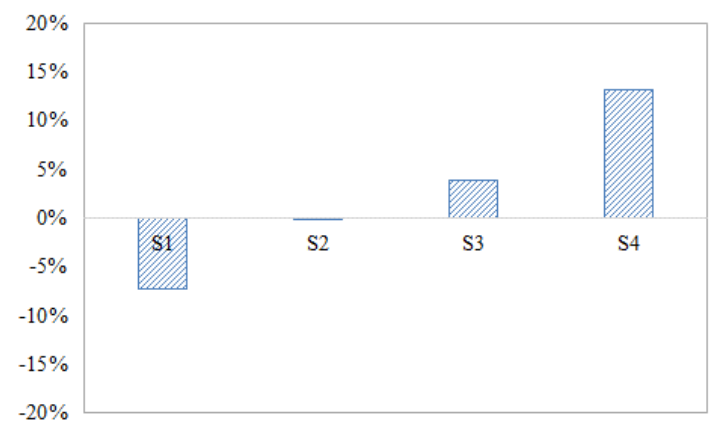

Figure 5. Relative error of estimated respect to measured effective conductivity

As it can be observed in Figure 5, the highest error was observed for the lightest sample, S4. This could be due to a non-negligible contribution of convection and radiation heat transfer when the porosity increases. This result was confirmed by the highest modified Rayleigh number and the lowest Planck number previously calculated for sample S4.

\section{CONCLUSIONS}

Innovative fibrous thermal insulation materials made of Merino wool were produced scouring and carding textile wastes discarded during the garment fabrication process by a local textile company. The fibers were treated with a natural binder solution made of chitosan.

Four samples of different density were prepared and characterized by measuring thermophysical properties including density, porosity, thermal conductivity, thermal diffusivity and volumetric specific heat.

Thermal conductivity was linearly related to density, i.e. porosity. A SEM analysis showed a fibrous structure of the woolen material characterized by a random distribution of cylindrical fibers.

A detailed evaluation of the heat transfer modes in the fibrous structure pointed out that contributions of convection and radiation are negligible. The only significant heat transfer mode is the conduction in a two phase structure: solid and gas phases. A simple model was proposed for the prediction of the effective thermal conductivity, based on the Parallel model.

Anyway, the analysis showed that the error in the estimation of the effective thermal conductivity could become unacceptable for very porous materials, when convection and radiation become important ways of heat transfer through the material.

\section{ACKNOWLEDGMENTS}

Authors wish address a special thanks to the Company Gordon Confezioni srl (Cassano, Italy) for supplying the raw 
materials and for the contribution given to this research.

The research was carried out within the National Interest Research project (PRIN) "SUSTAIN/ABLE - SimultaneoUs STructural And energetIc reNovAtion of BuiLdings through innovativE solutions" funded by Italian Ministery of University and Research.

\section{REFERENCES}

[1] Liu L, Li H, Lazzaretto A, Manente G, Tong C, Liu Q, Li N. (2016). The development history and prospects of biomass-based insulation materials for buildings Renew Sust Energ Rev 69: 912-932. https://doi.org/10.1016/j.rser.2016.11.140

[2] Asdrubali F, D'Alessandro F, Schiavoni S. (2015). A review of unconventional sustainable building insulation materials. $\quad$ SM\&T 4 : 1-17 https://doi.org/10.1016/j.susmat.2015.05.002

[3] Calkins M. (2009). Materials for sustainable sites defined. in Materials for Sustainable Sites: A Complete Guide to the Evaluation, Selection, and Use of Sustainable Construction Material. USA, CA: Wiley 1-12

[4] Sev A. (2008). How can the construction industry contribute to sustainable development? A Conceptual Framework. Sust Dev 17: 161-173. https://doi.org/10.1002/sd.373

[5] Sabapathy KA, Gedupudi S. (2019). Straw bale based constructions: Measurement of effective thermal transport properties. Constr Build Mater, 198: 182-194. https://doi.org/10.1016/j.conbuildmat.2018.11.256

[6] Madurwar MV, Ralegaonkar RV, Mandavgane SA. (2013). Application of agro-waste for sustainable construction materials: A review. Constr Build Mater vol. 38:

$872-878$.

https://doi.org/10.1016/j.conbuildmat.2012.09.011

[7] Liuzzi S, Stefanizzi P. (2016). Experimental study on hygrothermal performances of indoor covering materials. International Journal of Heat and Technology 34(2): S365-S370. https://doi.org/10.18280/ijht.34Sp0225

[8] Baccilieri F, Bornino R, Fotia A, Marino C, Nucara A, Pietrafesa M. (2016). Experimental measurements of the thermal conductivity of insulant elements made of natural materials: Preliminary results. International Journal of Heat and Technology 34(2): S413-S419. https://doi.org/10.18280/ijht.34S231.

[9] Rubino C, Liuzzi S, Stefanizzi P, Martellotta F. (2018). Textile wastes in building sector: A review. Modelling, Measurement and Control B 87(3): 172-179. https://doi.org/10.18280/mmc_b.870309.

[10] Wazna ME, Gounni A, Bouari AE, Alami ME, Cherkaoui O. (2019). Development, characterization and thermal performance of insulating nonwoven fabrics made from textile waste. J Ind Text 48(7): 1167-1183. https://doi.org/10.1177/1528083718757526

[11] Patnaik A, Mvubu M, Muniyasamy S, Botha A, Anandjiwala RD. (2015). Thermal and sound insulation materials from waste wool andrecycled polyester fibers and their biodegradation studies. Energ Buildings 92: 161-169. https://doi.org/10.1016/j.enbuild.2015.01.056

[12] Trajković D, Jordeva S, Tomovska E, Zafirova K. (2016). Polyester apparel cutting waste as insulation material. J $\begin{array}{llll}\text { Text I } & 108(7): & 1238-1245\end{array}$ https://doi.org/10.1080/00405000.2016.1237335
[13] Stankovic SB, Popovic D, Poparic GB. (2008). Thermal properties of textile fabrics made of natural and regenerated cellulose fibers. Polym Test 27(1): 41-48. https://doi.org/10.1016/j.polymertesting.2007.08.003

[14] Miettinen L, Kekalainen P, Turpeinen T, Hyvaluoma J, Merikoski J, Timonen J. (2012). Dependence of thermal conductivity on structural parameters in porous samples. $\begin{array}{llll}\text { AIP } & \text { Adv } & 021101 .\end{array}$ https://doi.org/10.1063/1.3676435

[15] Bankvall C. (1973). Heat transfer in fibrous materials. J Test Eva 1(3): 235-243. https://doi.org/10.1520/JTE10010J

[16] Tilioua A, Libessart L, Lassue S. (2018). Characterization of the thermal properties of fibrous insulation materials made from recycled textile fibers for building applications: Theoretical and experimental analyses. Appl Therm. Eng. 142: 56-67. https://doi.org/10.1016/j.applthermaleng.2018.06.071

[17] Schiavoni S, D'Alessandro F, Bianchi F, Asdrubali F. (2016). Insulation materials for the building sector: A review and comparative analysis. Renew Sust. Energ. Rev 62: 988-1011. https://doi.org/10.1016/j.rser.2016.05.045

[18] Tugnoli A, Moricone R, Scarponi GE, Cozzani V. (2019). Effective thermal conductivity of fibrous fireproofing materials. Int $\mathrm{J}$ Therm. Sci. 136: 107-120. https://doi.org/10.1016/j.ijthermalsci.2018.09.035.

[19] Mati-Baouche N, Baynast HD, Lebert A, Sun S, LopezMingo CJS, Leclaire P, Michaud P. (2014). Mechanical, thermal and acoustical characterizations of an insulating bio-based composite made from sunflower stalks particles and chitosan. Ind Crops Prod 58: 244-250. https://doi.org/10.1016/j.indcrop.2014.04.022

[20] Martellotta F, Cannavale A, Matteis VD, Ayr U. (2018). Sustainable sound absorbers obtained from olive pruning wastes and chitosan binder. Appl. Acoust. 141: 71-78. https://doi.org/10.1016/j.apacoust.2018.06.022

[21] Al-Homoud MS. (2005). Performance characteristics and practical applications of common building thermal insulation materials. Build Environ. 40(3): 353-366. https://doi.org/10.1016/j.buildenv.2004.05.013

[22] Wei K, Lv C, Chen M, Zhou X, Dai Z, Shen D. (2015). Development and performance evaluation of a new thermal insulation material from rice straw using high frequency hot-pressing Energ. Buildings 87: 116-122. https://doi.org/10.1016/j.enbuild.2014.11.026

[23] Barber RW, Emerson DR. (2006). Challenges in Modeling Gas-Phase Flow in Microchannels: From Slip to Transition. Heat Transfer Eng. 27(4): 3-12. https://doi.org/10.1080/01457630500522271

[24] Lurie SA, Solyaev YO, Lizunova DV, Rabinskiy LN, Bouznik VM, Menshykov O. (2017). Influence of mean distance between fibers on the effective gas thermal conductivity in highly porous fibrous materials Int J Heat Mass $\quad$ Transf. 109: 511-519. https://doi.org/10.1016/j.ijheatmasstransfer.2017.02.015

[25] Zhang WM, Meng G, Wei X. (2012). A review on slip models for gas microflows. Microfluid Nanofluid 13(6): 845-882. https://doi.org/10.1007/s10404-012-1012-9

[26] Zhang M, He M, Gu H, Huang A, Xiang W. (2018). Influence of pore distribution on the equivalent thermal conductivity of low porosity ceramic closed-cell foams. Ceram Int 44(16): 19319-19329. https://doi.org/10.1016/j.ceramint.2018.07.160 
[27] Incropera FP, Frank P, DeWitt DP. (2002). Fundamentals of heat and mass transfer. J. Wiley, New York.

[28] Silberstein A, Langlais C. (1990). Natural convection in light fibrous insulating materials with permeable interfaces: onset criteria and its effect on the thermal performances of the product. Journal of thermal insulation 14(1): https://doi.org/10.1177/109719639001400104

[29] Jackson GW, James DF. (1986). The permeability of fibrous porous media. Can J Chem Eng. 64: 364-374. https://doi.org/10.1002/cjce.5450640302

[30] Howell JR, Siegel R, Menguc MP. (2010). Thermal radiation heat transfer. Fifth Edition, CRC Press, New York.

[31] Lux J, Ahmadi A, Gobbé C, Delisée C. (2006). Macroscopic thermal properties of real fibrous materials: Volume averaging method and 3D image analysis. Int. J. Heat Mass Transf. 49(11-12): 1958-1973. https://doi.org/10.1016/j.ijheatmasstransfer.2005.09.038

[32] Gong L, Wang Y, Cheng X, Zhang R, Zhang H. (2014). A novel effective medium theory for modelling the thermal conductivity of porous materials. Int J Heat Mass Transf. 68:

295-298.

https://doi.org/10.1016/j.ijheatmasstransfer.2013.09.043

\section{NOMENCLATURE}

$c$

$d$

$d_{m}$

$f$

$g$

$k$

$k_{B}$

$l_{p}$ specific heat capacity, J. $\mathrm{kg}^{-1} \cdot \mathrm{K}^{-1}$

fibers diameter, $\mathrm{m}$

air collision diameter, $m$

volume fraction of solid, gravitational acceleration, $\mathrm{m} . \mathrm{s}^{-2}$ thermal conductivity, $\mathrm{W} \cdot \mathrm{m}^{-1} \cdot \mathrm{K}^{-1}$

Boltzmann's constant, J.K $\mathrm{K}^{-1}$

free path of photons, $\mathrm{m}$ $n$

$r$

$S$

$K$

$\mathrm{Kn}$

$N$

$P$

$R a^{*}$

$T$

\section{Greek symbols}

$\alpha$

$\beta$

$\delta$

$\varepsilon$

$\lambda$

$\mu$

$\rho$

$\sigma$

$\Delta T$

\section{Subscripts}

$\begin{array}{ll}\text { air } & \text { air value } \\ \text { bulk } & \text { bulk value } \\ \text { c } & \text { conduction } \\ \text { conv } & \text { natural convection } \\ e & \text { effective value } \\ f & \text { fibers } \\ g & \text { gas } \\ r & \text { radiation } \\ \text { true } & \text { true value }\end{array}$

refractive index, radius of the fibers, $m$ thickness of material, $\mathrm{m}$ permeability, $\mathrm{m}^{2}$

Knudsen number, -

Planck number, -

pressure, $\mathrm{Pa}$

modified Rayleigh number, temperature, $\mathrm{K}$

thermal diffusivity, $\mathrm{m}^{2} . \mathrm{s}^{-1}$ volumetric thermal expansion coefficient of air, $\mathrm{K}^{-1}$ characteristic length, $\mathrm{m}$ porosity, molecular free path, $\mathrm{m}$ dynamic viscosity, $\mathrm{kg} \cdot \mathrm{m}^{-1} \cdot \mathrm{s}^{-1}$ bulk density, $\mathrm{kg} \cdot \mathrm{m}^{-3}$ Stefan-Boltzmann's constant, W. $\mathrm{m}^{-2} \cdot \mathrm{K}^{-4}$ temperature difference, $\mathrm{K}$ 